\title{
Preface to the Special Issue: Application of Synchrotron Radiation in Materials Research
}

\author{
Qiaodan $\mathrm{Hu}^{1}$
}

Published online: 24 December 2021

(c) The Chinese Society for Metals (CSM) and Springer-Verlag GmbH Germany, part of Springer Nature 2021

Synchrotron radiation was observed for the first time by Haber et al. at General Electric in the United States in 1947 in a $70-\mathrm{MeV}$ electron synchrotron. The development of synchrotron radiation light source has gone through four generations since the 1960s. Dozens of synchrotron radiation facilities (SRFs) for scientific research have been built around the world, such as Spring-8 in Japan, Advanced Photon Source (APS) in the United States and ESRF in Europe. In China, the first- and the second-generation synchrotron radiation light sources were constructed in the 1990s in Beijing and Hefei, known as Beijing Synchrotron Radiation Facility (BSRF) and Hefei national synchrotron radiation laboratory (NSRL), respectively. The third-generation SRF, Shanghai synchrotron radiation Facility (SSRF), was built in 2009, and the fourth generation, a high-energy photon source, is under construction in Beijing and is expected to be operational in 2025 .

With the advantages of broad frequency spectrum, high brilliance, high degree of coherence, as well as being highly collimated and polarized, synchrotron radiation provides a novel experimental tool for scientific research, and has made significant contributions to the development of related scientific and technological fields. From viewpoint of methodology, synchrotron radiation is usually categorized into three techniques, i.e., X-ray imaging, X-ray scattering and diffraction, and X-ray spectroscopy. Undoubtedly, synchrotron radiation will play a more and more important role in understanding the structure-property relationship of existing materials, exploring new materials, as well as visualizing and revealing what happens during materials processing.

In this special issue, recent progresses on the application of synchrotron radiation in materials research are reported. Thirteen insightful papers have been accepted for final publication in the issue. Among these papers, three review articles by Fan et al., Shuai et al., and Zeng et al. carefully address the development of synchrotron radiation technology in research on metallic materials and additive manufacturing. The research papers, on the other hand, report applications of synchrotron radiation in exploring the microstructural evolution both in 2D and 3D, crystallization kinetics, phase transformations, etc., covering a wide range of materials including aluminum alloys, Ni-based superalloy, metallic glass, composites and superconductors. The subjects of the papers cover the recent major progresses of applications of synchrotron radiation in materials characterizations.

We sincerely thank all the authors for their noteworthy contributions and all the reviewers for their critical and constructive assessment. We also appreciate the helpful assistance from the editors and the publishing team of Acta Metallurgica Sinica (English Letters) in organizing and publishing this special issue. We hope that this special issue will provide the readers useful and up-to-date information on the current state of synchrotron radiation and its application in materials research.

Qiaodan $\mathrm{Hu}$

qdhu@sjtu.edu.cn

1 Shanghai Key Laboratory of Materials Laser Processing and Modification, School of Materials Science and Engineering, Shanghai Jiao Tong University, Shanghai 200240, China 


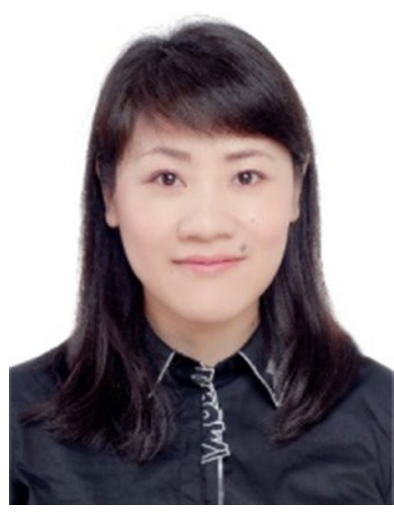

Dr. Qiaodan Hu is a professor of Materials Science and Engineering at Shanghai Jiao Tong University (SJTU), China. She was awarded Outstanding Young Scholar by National Natural Science Foundation of China and Young Yangtze River Scholar by the Ministry of Education. Dr. $\mathrm{Hu}$ is currently serving as a committee member of several academic organizations, including "Executive Director of the Youth Working Committee of Chinese Materials Research Society",
"Member/Deputy Secretary General of Solidification Science and Technology Branch of Chinese Materials Research Society", "Member of Physical and Chemical Metallurgy Process Branch of The Chinese Society for Metals", the youth editor of "Acta Metallurgica Sinica", "Transactions of Nonferrous Metals Society of China", "International Journal of Minerals, Metallurgy and Materials", etc. Her research mainly focuses on solidification theory, technology and application with expertise in probing the melt structure, nucleation and polymorphic selection behavior, and crystal growth mechanism of a wide range of metallic and ceramic materials by synchrotron radiation technology. Dr. Hu has published $\sim 80$ peer-reviewed journal papers on solidification and synchrotron radiation and will continue to dedicate her research efforts toward further improvement and innovation in this field. 